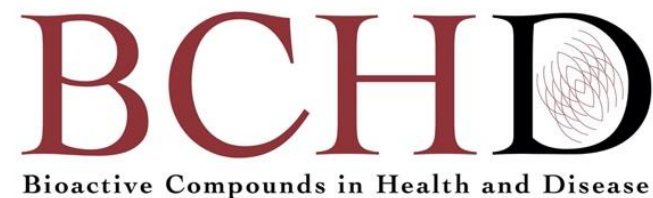

\title{
Vitamin D and COVID-19: Partial Evidence
}

\section{Mona Boaz}

Ariel University, Ariel, Israel

Corresponding Author: Prof. Mona Boaz, RD, PhD, Chair, Department of Nutrition Sciences, Ariel University, Ramat HaGolan St 65, Ariel, Israel

Submission Date: March 26 ${ }^{\text {th }}, 2021$; Acceptance Date: March 26 ${ }^{\text {th }}, 2021$; Publication Date: March $30^{\text {th }}, 2021$

Please cite this article as: Boaz M. Vitamin D and COVID-19: partial evidence. Bioactive Compounds in Health and Disease 2021. 4(3): 40-44. DOI: https://www.doi.org/10.31989/bchd.v4i3.796

\section{Vitamin D and COVID-19: Partial Evidence}

The COVID-19 pandemic, now in its second year, has created challenges for health maintenance. Lockdowns, working from home and social distancing have all created changes to the way we live, increasing stress, anxiety and depression and impacting our lifestyle habits such as diet and exercise [1]. This may be particularly concerning considering the association between obesity and adverse outcomes of COVID infection including hospitalization, invasive ventilation, and death [2].

While vaccines are now available, they were not an option earlier in the pandemic, and researchers and clinicians alike sought interventions to prevent infection and reduce risk of adverse outcomes. Among these are nutrition supplementation with vitamin D. This nutrient plays an important role in maintaining the integrity of the immune system; however, it is essential to examine its performance on a population level to assess its efficacy, and to consider findings in the context of the hierarchy of evidence.

\section{Vitamin D and Covid - Mechanisms}

Technically a hormone rather than a vitamin, vitamin D can be generated in the skin when light energy is absorbed by the precursor molecule [3]. Vitamin D can also be absorbed from food, such as fatty fish and fortified foods [4]. Well known for its role in bone metabolism, vitamin $D$ is also essential for innate immune function [5].

Innate immunity, the body's initial and immediate response to pathogenic challenge, involves the complement system, and includes neutrophil and macrophage antimicrobial responses. Monocytes and macrophages can phagocytose pathogens [6]. Additionally, they can sense pathogen-associated molecular patterns from bacteria, parasites, fungi and viruses [7]. Vitamin D has been shown to enhance innate immunity by 
inducing anti-viral peptides including NOD2/CARD15defensin $\beta 2$ [8] and cathelicidin [9], which improves mucosal defenses.

In addition to the role of vitamin $D$ in innate immunity, deficient serum vitamin $D$ is associated with both increased autoimmunity [10] and increased susceptibility to infection [11]. Vitamin D has also been shown to inhibit cytokine storms [12].

\section{Seasonality}

The seasonal stimulus hypothesis proposes an explanation for the winter outbreak pattern of influenza A. According to this theory, influenza A outbreaks correspond to seasonal reduction in sun exposure, leading to a reduction in vitamin D levels, and a suppression of immune response [13]. While the seasonality of COVID-19 has not yet been established, associations with sun exposure have been reported. An ecologic study used proximity to the equator as an estimation of mean sun exposure and correlated this to COVID-19 mortality in 88 countries. The association was attributed to alterations in Vitamin D levels associated with sun exposure [14]. This concept is further supported by observations regarding increased COVID-19 mortality among individuals with darker skin [15]. Indeed, it has been shown that vitamin $\mathrm{D}$ deficiency was almost 9 times more prevalent among lower non-Hispanic African Americans than non-Hispanic Caucasians [16]. Among elderly patients, who fared particularly badly during the pandemic, pre-existing, age-associated immune dysregulation coupled with vitamin D deficiency were mechanisms proposed to explain the increased COVID-19 mortality rates in this group [17]. It is noteworthy that sun exposure among the elderly, particularly those who live in long-term care facilities, is less than that of other individuals [18].

\section{Case series and cross-sectional studies}

Investigators studied four patients with vitamin D deficiency diagnosed with COVID-19 in April 2020 and treated them with either low dose $(1000 \mathrm{I} / \mathrm{U})$ or high dose $(50,000 \mathrm{I} / \mathrm{U})$ vitamin $\mathrm{D}$ for five days. Patients who received high dose vitamin $D$ achieved normalization of vitamin D levels, shorter hospital stay, lower supplemental oxygen requirement and reduced blood markers of inflammation [19]. While case series are not actually research (no hypothesis is tested), this encouraging report no doubt encouraged further research in the use of vitamin $D$ as a therapeutic in COVID-19.

Cross sectional studies measure exposure and outcome simultaneously. This study design was ideal in the early phase of the COVID-19 pandemic when longitudinal data were not available. In one such study, investigators noted than hospitalized individuals with vitamin D deficiency were 4.6 times as likely to be positive for COVID-19 as their nonvitamin D deficient counterparts. This association persisted after controlling for potential confounders including age, sex and comorbidities [20] Consistent with these findings was a study from China in which vitamin D deficiency, defined as $<30 \mathrm{nmol} / \mathrm{L}$, increased risk of COVID-19 severity by a factor of 2.72 . This association remained robust even after controlling for age and sex [21].

These findings support the notion of an association between vitamin D deficiency and risk for COVID-19 and disease severity but cannot establish causality. By definition, when exposure and outcome are contemporaneously measured, temporality 
cannot be established, a prerequisite to determining causality.

\section{Retrospective studies}

Several retrospective studies have been reported on the association between vitamin D and COVID-19. One study compared vitamin D levels in COVID-19 patients by vital status (recovered vs. deceased). An overall difference in vitamin D levels were not identified; however, among individuals with severe COVID-19, vitamin D levels were lower among those who died than among those who ultimately recovered [22]. In a large retrospective study in the US, vitamin D levels ascertained prior to COVID-19 testing did not differ between white individuals who tested positive or negative for COVID 19; however, among Black participants, those with vitamin D levels $<40 \mathrm{ng} / \mathrm{mL}$ were at increased risk of a positive COVID19 test [23]. Another retrospective study conducted in individuals enrolled in a New York City Health System and positive for COVID-19 found that vitamin $D$ deficiency, defined as levels $<20 \mathrm{ng} / \mathrm{mL}$, increased risk for oxygen support therapy, but not for hospitalization or death [24].

Findings of retrospective studies suggest that the association between vitamin D levels and COVID19 outcomes are perhaps more nuanced than crosssectional studies might suggest. It becomes clear that the association might be conditional on patient characteristics such as race. Further, it is important to remember that retrospective studies look backward in time to ascertain exposure status and is thus limited to individuals with a measure of vitamin $D$ status prior to ascertainment of COVID-19 status. Such individuals may be at different risk for COVID-19 than others due to comorbidities or medical followup. This would impact the external validity of the findings.

\section{Prospective studies}

Prospective studies begin with the exposure, in this case vitamin D levels, and follow participants forward in time to ascertain outcome. Because they begin with the exposure, temporality can be firmly established, permitting determination of causality.

One prospective study of 410 patients admitted for COVID-19 categorized vitamin D levels as deficient ( $<20 \mathrm{ng} / \mathrm{mL}$ ) or not based on blood tests obtained at hospital admission. These admission serum vitamin D levels did not correlate with inflammatory markers or clinical outcomes, including death [25]. By contrast, another prospective study found that habitual vitamin D supplementation was significantly associated with reduced risk of subsequent COVID-19 infection; however, blood vitamin D levels and genetically predicted blood vitamin D levels were not associated with COVID-19 risk. It cannot be ruled out that self-supplementation with vitamin D represents some other behavior associated with reduced COVID19 risk, because vitamin D levels and predicted vitamin D levels did not differ by vitamin D supplementation [26].

\section{Clinical trials}

Compared to observational studies, clinical trials provide convincing evidence for the efficacy for interventions. Randomization to treatment reduces confounding and serves to isolate the influence of the exposure, which may be therapeutic or preventive.

In a clinical trial, 240 individuals were randomized to treatment with a single dose of 200000 IU of vitamin D3 or placebo. The trial was designed to examine the efficacy of this intervention on duration of hospital stay, defined as the number of days from admission to discharge. This outcome did not differ by treatment assignment. While vitamin D levels increased dramatically in the active treatment 
group vs. placebo, differences in secondary endpoints, including mortality, need for mechanical ventilation or admission to intensive care unit, did not differ by vitamin D treatment [27].

In a clinical trial described as a pilot study, 50 patients were randomized to treatment with oral calcifediol (0.532 $\mathrm{mg}$ ) beginning on the day of admission, then on days 3 and 7, and weekly thereafter. The control group did not receive this treatment, nor did they receive a placebo or comparator treatment. Of the 50 patients treated with calcifediol, one required admission to the ICU (2\%), compared to 13 of the 26 patients who received no treatment. This represents a relative reduction of ICU admission risk of $98 \%$. This association remained robust after controlling for comorbid type 2 diabetes or hypertension. No deaths were observed in the calcifediol group, while two deaths were observed in the control group among the 13 patients who had been admitted to ICU [28].

\section{Recommendations}

Good nutrition is essential for adequate immune function, supporting resistance to infectious disease and reducing adverse outcomes in the event of illness. Associated with well-being, good nutrition may facilitate better coping skills and reduce anxiety during lockdowns [29].

Adequate vitamin D nutriture in the context of a healthy diet is undoubtedly worth emphasizing, particularly in individuals with demonstrated vitamin D deficiency. It is important to remember, however, that indiscriminate supplementation is not without potential harm; indeed, hypervitaminosis D can occur [30]. While there are several mechanisms through which vitamin D influences the human immune system, there is not adequate evidence of its performance in human populations with COVID-19.
Well-designed, adequately powered clinical trials are needed to examine the role of this nutrient in both prevention and treatment of COVID-19.

\section{REFERENCES}

1. Al-Domi H, Al-Dalaeen A, Al-Rosan S, Batarseh $\mathrm{N}$, Nawaiseh $\mathrm{H}$. Healthy nutritional behavior during COVID19 lockdown: A cross-sectional study. Clin Nutr ESPEN. 2021;42:132-137.

2. Kompaniyets L, Goodman AB, Belay B, Freedman DS, Sucosky MS, Lange SJ, Gundlapalli AV, Boehmer TK, Blanck HM. Body Mass Index and Risk for COVI D-19Related Hospitalization, Intensive Care Unit Admission, Invasive Mechanical Ventilation, and Death - United States, March-December 2020. MMWR Morb Mortal Wkly Rep. 2021;70:355-361..

3. Bikle DD. Vitamin D metabolism, mechanism of action, and clinical applications. Chem Biol. 2014; 21:319-29.

4. Lehmann $U$, Gjessing HR, Hirche F, Mueller-Belecke A, Gudbrandsen OA, Ueland PM, Mellgren G, Lauritzen L, Lindqvist $H$, Hansen AL, Erkkilä AT, Pot GK, Stangl GI, Dierkes J. Efficacy of fish intake on vitamin D status: a meta-analysis of randomized controlled trials. Am J Clin Nutr. 2015;102:837-47.

5. Smith TJ, McClung JP. Nutrition, Immune Function, and Infectious Disease. Med J (Ft Sam Houst Tex). 2021;(PB 8-21-01/02/03):133-136.

6. Caponegro MD, Thompson KK, Tayyab M, Tsirka SE. A Rigorous Quantitative Approach to Analyzing Phagocytosis Assays. Bio Protoc. 2020;10:e3698.

7. Kawai T, Akira S. The role of pattern-recognition receptors in innate immunity: update on Toll-like receptors. Nat Immunol. 2010;11:373-84.

8. Wang T.-T., Dabbas B., Laperriere D., Bitton A.J., Soualhine H., Tavera-Mendoza L.E. Direct and indirect induction by 1,25-dihydroxyvitamin D3 of the NOD2/CARD15-defensin $\beta 2$ innate immune pathway defective in Crohn disease. J Biol Chem. 2010;85:22272231.

9. Gombart A.F., Borregaard N., Koeffler H.P. Human cathelicidin antimicrobial peptide (CAMP) gene is a direct target of the vitamin $D$ receptor and is strongly up-regulated in myeloid cells by 1,25-dihydroxyvitamin D3. FASEB J. 2005;19:1067-1077.

10. Fang $F$, Chai $Y$, Wei H, Wang K, Tan L, Zhang W, Fan Y, Li $F$, Shan Z, Zhu M. Vitamin D deficiency is associated with thyroid autoimmunity: results from an epidemiological survey in Tianjin, China. Endocrine. 2021. doi: 10.1007/s12020-021-02688-z. Epub ahead of print. PMID: 33759075.

11. Muthuvattur Pallath $M$, Ahirwar AK, Chandra Tripathi $S$, Asia P, Sakarde A, Gopal N. COVID-19 and nutritional deficiency: a review of existing knowledge. Horm Mol Biol Clin Investig. 2021. doi: 10.1515/hmbci-2020-0074. Epub ahead of print. PMID: 33544528.

12. Jain SK, Micinski D, Parsanathan R. I-Cysteine Stimulates the Effect of Vitamin D on Inhibition of Oxidative Stress, IL-8, and MCP-1 Secretion in High Glucose Treated Monocytes. J Am Coll Nutr. 2021 Feb 17:1-6. doi: 10.1080/07315724.2020.1850371. Epub ahead of print. PMID: 33596158.

13. Cannell JJ, Vieth R, Umhau JC, Holick MF, Grant WB, Madronich S, Garland CF, Giovannucci E. Epidemic influenza and vitamin D. Epidemiol Infect. 2006;134:1129-40.

14. Whittemore PB. COVID-19 fatalities, latitude, sunlight, and vitamin D. Am J Infect Control. 2020; 48:1042-1044. 
15. Ayoubkhani D, Nafilyan V, White C, Goldblatt $P$, Gaughan C, Blackwell L, Rogers N, Banerjee A, Khunti K, Glickman M, Humberstone B, Diamond I. Ethnicminority groups in England and Wales-factors associated with the size and timing of elevated COVID19 mortality: a retrospecti

16. Herrick KA, Storandt RJ, Afful J, Pfeiffer CM, Schleicher RL, Gahche JJ, Potischman N. Vitamin D status in the United States, 2011-2014. Am J Clin Nutr. 2019;110:150-157.

17. Meftahi GH, Jangravi Z, Sahraei H, Bahari Z. The possible pathophysiology mechanism of cytokine storm in elderly adults with COVID-19 infection: the contribution of "inflame-aging". Inflamm Res. 2020; 69:825-839.

18. Samefors M, Tengblad A, Östgren CJ. Sunlight Exposure and Vitamin D Levels in Older People- An Intervention Study in Swedish Nursing Homes. J Nutr Health Aging. 2020;24:1047-1052.

19. Ohaegbulam KC, Swalih M, Patel P, Smith MA, Perrin R. Vitamin D Supplementation in COVID-19 Patients: A Clinical Case Series. Am J Ther. 2020;27:e485-e490.

20. Katz J, Yue S, Xue W. Increased risk for COVID-19 in patients with vitamin D deficiency. Nutrition. 2021;84:111106.

21. Luo X, Liao Q, Shen Y, Li H, Cheng L. Vitamin D Deficiency Is Associated with COVID-19 Incidence and Disease Severity in Chinese People [corrected]. J Nutr. 2021;151:98-103.

22. Tehrani S, Khabiri N, Moradi H, Mosavat MS, Khabiri SS Evaluation of vitamin D levels in COVID-19 patients referred to Labafinejad hospital in Tehran and its relationship with disease severity and mortality. Clin Nutr ESPEN. 2021;42:313-317.

23. Meltzer DO, Best TJ, Zhang $\mathrm{H}$, Vokes $\mathrm{T}$, Arora VM, Solway J. Association of Vitamin D Levels, Race/Ethnicity, and Clinical Characteristics With COVID19 Test Results. JAMA Netw Open. 2021;4:e214117.
24. Gavioli EM, Miyashita H, Hassaneen O, Siau E. An Evaluation of Serum 25-Hydroxy Vitamin D Levels in Patients with COVID-19 in New York City. J Am Coll Nutr. 2021:1-6.

25. Jevalikar G, Mithal A, Singh A, Sharma R, Farooqui KJ, Mahendru S, Dewan A, Budhiraja S. Lack of association of baseline 25-hydroxyvitamin $D$ levels with disease severity and mortality in Indian patients hospitalized for COVID-19. Sci Rep. 2021;11:6258

26. Ma H, Zhou T, Heianza Y, Qi L. Habitual use of vitamin D supplements and risk of coronavirus disease 2019 (COVID-19) infection: a prospective study in UK Biobank. Am J Clin Nutr. 2021:nqaa381. doi: 10.1093/ajcn/nqaa381. Epub ahead of print.

27. Murai IH, Fernandes AL, Sales LP, Pinto AJ, Goessler KF, Duran CSC, Silva CBR, Franco AS, Macedo MB, Dalmolin $\mathrm{HHH}$, Baggio J, Balbi GGM, Reis BZ, Antonangelo L, Caparbo VF, Gualano B, Pereira RMR. Effect of a Single High Dose of Vitamin D3 on Hospital Length of Stay in Patients With Moderate to Severe COVID-19: A Randomized Clinical Trial. JAMA. 2021;325:1053-1060..

28. Entrenas Castillo $M$, Entrenas Costa LM, Vaquero Barrios JM, Alcalá Díaz JF, López Miranda J, Bouillon R, Quesada Gomez JM. "Effect of calcifediol treatment and best available therapy versus best available therapy on intensive care unit admission and mortality among patients hospitalized for COVID-19: A pilot randomized clinical study". J Steroid Biochem Mol Biol. 2020;203:105751..

29. Kaufman-Shriqui V, Navarro DA, Raz O, Boaz M. Multinational dietary changes and anxiety during the coronavirus pandemic-findings from Israel. Isr J Health Policy Res. 2021;10:28.

30. Ghauri MI, Bareeqa SB, Riaz A, Kumar A. Redundancy Is of No Good; latrogenic Hypervitaminosis D: A Rare Case of Persistent Vomiting Due to Hypercalcemia. Clin Med Insights Case Rep. 2019;12:1179547619828688. 